PROCEEDINGS OF THE

AMERICAN MATHEMATICAL SOCIETY

Volume 139, Number 12, December 2011, Pages 4199-4206

S 0002-9939(2011)10901-8

Article electronically published on April 21, 2011

\title{
ON STABLY FREE MODULES OVER LAURENT POLYNOMIAL RINGS
}

\author{
ABED ABEDELFATAH
}

(Communicated by Harm Derksen)

\begin{abstract}
We prove constructively that for any finite-dimensional commutative ring $R$ and $n \geq \operatorname{dim}(R)+2$, the group $\mathrm{E}_{n}\left(R\left[X, X^{-1}\right]\right)$ acts transitively on $\operatorname{Um}_{n}\left(R\left[X, X^{-1}\right]\right)$. In particular, we obtain that for any finite-dimensional ring $R$, every finitely generated stably free module over $R\left[X, X^{-1}\right]$ of rank $>\operatorname{dim} R$ is free; i.e., $R\left[X, X^{-1}\right]$ is $(\operatorname{dim} R)$-Hermite.
\end{abstract}

\section{INTRODUCTION}

We denote by $R$ a commutative ring with unity and $\mathbb{N}$ the set of non-negative integers. $\operatorname{Um}_{n}(R)$ is the set of unimodular rows of length $n$ over $R$, that is, all $\left(x_{0}, \ldots, x_{n-1}\right) \in R^{n}$ such that $x_{0} R+\cdots+x_{n-1} R=R$. If $u, v \in \operatorname{Um}_{n}(R)$ and $G$ is a subgroup of $\mathrm{GL}_{n}(R)$, we write $u \sim_{\mathrm{G}} v$ if there exists $g$ in $G$ such that $v=u g$. Recall that $\mathrm{E}_{n}(R)$ denotes the subgroup of $\mathrm{GL}_{n}(R)$, generated by all $\mathrm{E}_{i j}(a):=I_{n}+a e_{i j}$ (where $i \neq j, a \in R$ and $e_{i j}$ denotes the $(n \times n)$-matrix whose only non-zero entry is 1 on the $(i, j)$ entry). We abbreviate the notation $u \sim_{\mathrm{E}_{n}(R)} v$ to $u \sim_{\mathrm{E}} v$. We say that a ring $R$ is Hermite (resp. $d$-Hermite) if any finitely generated stably free $R$-module (resp., any finitely generated stably free $R$-module of rank $>d$ ) is free.

In [6], A. A. Suslin proved:

Theorem 1.1 (A. A. Suslin). If $R$ is a Noetherian ring and

$$
A=R\left[X_{1}^{ \pm 1}, \ldots, X_{k}^{ \pm 1}, X_{k+1}, \ldots, X_{n}\right],
$$

then for $n \geq \max (3, \operatorname{dim}(R)+2)$ the group $\mathrm{E}_{n}(A)$ acts transitively on $\operatorname{Um}_{n}(A)$.

In particular we obtain that $\mathrm{E}_{n}\left(R\left[X, X^{-1}\right]\right)$ acts transitively on $\operatorname{Um}_{n}\left(R\left[X, X^{-1}\right]\right)$ for any Noetherian $\operatorname{ring} R$, where $n \geq \max (3, \operatorname{dim}(R)+2)$. In [7, I. Yengui proved:

Theorem 1.2 (I. Yengui). Let $R$ be a ring of dimension $d, n \geq d+1$, and let $f \in \mathrm{Um}_{n+1}(R[X])$. Then there exists $E \in \mathrm{E}_{n+1}(R[X])$ such that $f \cdot E=e_{1}$.

In this article we generalize by proving:

Received by the editors May 29, 2010 and, in revised form, September 25, 2010 and October 19, 2010.

2010 Mathematics Subject Classification. Primary 13Axx; Secondary 13A50.

Key words and phrases. Stably free modules, Hermite rings, unimodular rows, Laurent polynomial rings, constructive mathematics.

(C)2011 American Mathematical Society Reverts to public domain 28 years from publication 
Theorem 1.3. For any finite-dimensional ring $R, \mathrm{E}_{n}\left(R\left[X, X^{-1}\right]\right)$ acts transitively on $\operatorname{Um}_{n}\left(R\left[X, X^{-1}\right]\right)$, where $n \geq \operatorname{dim}(R)+2$.

This gives a positive answer to Yengui's question (Question 9 of [7]). The proof we give is a close adaptation of Yengui's proof of the Laurent case.

\section{PReliminary RESUlts ON UNimOdUlar ROWS}

A. A. Suslin proved in [6], that if $f=\left(f_{0}, \ldots, f_{n}\right) \in \operatorname{Um}_{n+1}(R[X])$, where $f_{1}$ is unitary and $n \geq 1$, then there exists $w \in \mathrm{SL}_{2}(R[X]) \cdot \mathrm{E}_{n+1}(R[X])$ such that $f \cdot w=e_{1}$. In fact, this theorem is a crucial point in his proof of Serre's conjecture. R. A. Rao generalized in [4], Corollary 2. 5] by proving:

Theorem 2.1 (R. A. Rao [4]). Let $f=\left(f_{0}, \ldots, f_{n}\right) \in \operatorname{Um}_{n+1}(R[X])$, where $n \geq 2$. If some $f_{i}$ is unitary, then $f$ is completable to a matrix in $\mathrm{E}_{n}(R[X])$.

Recall that the boundary ideal of an element $a$ of a ring $R$ is the ideal $\mathcal{I}(a)$ of $R$ generated by $a$ and all $y \in R$ such that $a y$ is nilpotent. Moreover, $\operatorname{dim} R \leq d \Leftrightarrow$ $\operatorname{dim}(R / \mathcal{I}(a)) \leq d-1$ for all $a \in R[3$.

Theorem 2.2 ([2], Theorem 2.4). Let $R$ be a ring of dimension $\leq d$ and $a=$ $\left(a_{0}, \ldots, a_{n}\right) \in \mathrm{Um}_{n+1}(R)$ where $n \geq d+1$. Then there exist $b_{1}, \ldots, b_{n} \in R$ such that

$$
\left\langle a_{1}+b_{1} a_{0}, \ldots, a_{n}+b_{n} a_{0}\right\rangle=R .
$$

In fact, we can obtain a stronger result if $f \in \operatorname{Um}_{n+1}\left(R_{S}\right)$, where $S$ is a multiplicative subset of $R$ :

Proposition 2.3. Let $S$ be a multiplicative subset of $R$ such that $S^{-1} R$ has dimensiond. Let $\left(a_{0}, \ldots, a_{n}\right) \in M_{n+1}(R)$ be a row such that $\left(\frac{a_{0}}{1}, \ldots, \frac{a_{n}}{1}\right) \in \operatorname{Um}_{n+1}\left(S^{-1} R\right)$, where $n>d$. Then there exist $b_{1}, \ldots, b_{n} \in R$ and $s \in S$ such that

$$
s \in\left(a_{1}+b_{1} a_{0}\right) R+\cdots+\left(a_{n}+b_{n} a_{0}\right) R .
$$

Proof. By induction on $d$, if $d=0$, then $R_{S} / \mathcal{I}\left(\frac{a_{n}}{1}\right) \cong(R / J)_{\bar{S}}$ is trivial, where $\bar{S}=\{s+J \mid s \in S\}, J=i^{-1}\left(\mathcal{I}\left(\frac{a_{n}}{1}\right)\right)$, and $i: R \rightarrow R_{S}$ is the natural homomorphism. So $1 \in\left\langle\frac{a_{n}}{1}, \frac{b_{n}}{1}\right\rangle$ in $R_{S}$, where $b_{n} \in R$ and $\frac{a_{n} b_{n}}{1}$ is nilpotent. Since $1 \in\left\langle\frac{a_{1}}{1}, \ldots, \frac{a_{n-1}}{1}, \frac{a_{n}}{1}, \frac{b_{n} a_{0}}{1}\right\rangle$, so by [2], Lemma 2.3], $1 \in\left\langle\frac{a_{1}}{1}, \ldots, \frac{a_{n-1}}{1}, \frac{a_{n}+b_{n} a_{0}}{1}\right\rangle$; i.e., there exist $s \in S$ such that $s \in a_{1} R+\cdots+a_{n-1} R+\left(a_{n}+b_{n} a_{0}\right) R$.

Assume now that $d>0$. By the induction assumption with respect to the ring $R_{S} / \mathcal{I}\left(\frac{a_{n}}{1}\right) \cong(R / J)_{\bar{S}}$ we can find $\bar{b}_{1}, \ldots, \bar{b}_{n-1} \in R / J$ such that

$$
\left\langle\frac{\bar{a}_{1}+\bar{b}_{1} \bar{a}_{0}}{\overline{1}}, \ldots, \frac{\bar{a}_{n-1}+\bar{b}_{n-1} \bar{a}_{0}}{\overline{1}}\right\rangle=(R / J)_{\bar{S}} .
$$

So $\left\langle\overline{\frac{a_{1}+b_{1} a_{0}}{1}}, \ldots, \overline{\frac{a_{n-1}+b_{n-1} a_{0}}{1}}\right\rangle=R_{S} / \mathcal{I}\left(\frac{a_{n}}{1}\right)$. This means that

$$
\left\langle\frac{a_{1}+b_{1} a_{0}}{1}, \ldots, \frac{a_{n-1}+b_{n-1} a_{0}}{1}, \frac{a_{n}}{1}, \frac{b_{n}}{1}\right\rangle=R_{S}
$$

where $\frac{a_{n} b_{n}}{1}$ is nilpotent. So by [[2], Lemma 2.3],

$$
\left\langle\frac{a_{1}+b_{1} a_{0}}{1}, \ldots, \frac{a_{n-1}+b_{n-1} a_{0}}{1}, \frac{a_{n}+b_{n} a_{0}}{1}\right\rangle=R_{S} .
$$


Let $f \in \operatorname{Um}_{n+1}(R[X])$, where $n \geq \frac{d}{2}+1$, with $R$ a local ring of dimension d. M. Roitman's argument in [[5], Theorem 5], shows how one could decrease the degree of all but one (special) co-ordinate of $f$. In the absence of a monic polynomial as a co-ordinate of $f$ he uses a Euclid's algorithm and this is achieved via the following:

Lemma 2.4 (M. Roitman, [5], Lemma 1). Let $\left(x_{0}, \ldots, x_{n}\right) \in \operatorname{Um}_{n+1}(R), n \geq 2$, and let $t$ be an element of $R$ which is invertible $\bmod \left(R x_{0}+\cdots+R x_{n-2}\right)$. Then

$$
\left(x_{0}, \ldots, x_{n}\right) \sim_{\mathrm{E}_{n+1}(R)}\left(x_{0}, \ldots, t^{2} x_{n}\right) \sim_{\mathrm{E}_{n+1}(R)}\left(x_{0}, \ldots, t x_{n-1}, t x_{n}\right) .
$$

\section{The MAIN RESUlts}

Definitions 3.1. Let $f \in R\left[X, X^{-1}\right]$ be a nonzero Laurent polynomial. We denote $\operatorname{deg}(f)=\operatorname{hdeg}(f)-\operatorname{ldeg}(f)$, where $\operatorname{hdeg}(f)$ and $\operatorname{ldeg}(f)$ denote respectively the highest and the lowest degree of $f$.

Let hc $(f)$ and $\operatorname{lc}(f)$ denote respectively the coefficients of the highest and the lowest degree term of $f$. An element $f \in R\left[X, X^{-1}\right]$ is called doubly unitary if $\mathrm{hc}(f), \operatorname{lc}(f) \in U(R)$.

For example, $\operatorname{deg}\left(X^{-3}+X^{2}\right)=5$.

Lemma 3.2. Let $f_{1}, \ldots, f_{n} \in R\left[X, X^{-1}\right]$ such that hdeg $\left(f_{i}\right) \leq k-1, \operatorname{ldeg}\left(f_{i}\right) \geq-m$ for all $1 \leq i \leq n$. Let $f \in R\left[X, X^{-1}\right]$ with $\operatorname{hdeg}(f)=k$, $\operatorname{ldeg}(f) \geq-m$, where $k, m \in \mathbb{N}$. Assume that $\mathrm{hc}(f) \in U(R)$ and the coefficients of $f_{1}, \ldots, f_{n}$ generate the ideal (1) of $R$. Then $I=\left\langle f_{1}, \ldots, f_{n}, f\right\rangle$ contains a polynomial $h$ of hdeg $(h)=k-1$, $\operatorname{ldeg}(h) \geq-m$ and $\mathrm{hc}(h) \in U(R)$.

Proof. Since $X^{m} f_{1}, \ldots, X^{m} f_{n}, X^{m} f \in R[X]$, by [1], $\S 4$, Lemma 1(b)], I contains a polynomial $h_{1} \in R[X]$ of degree $m+k-1$ which is unitary. So $h=X^{-m} h_{1} \in I$ of $\operatorname{hdeg}(h)=k-1, \operatorname{ldeg}(h) \geq-m$ and $\operatorname{hc}(h) \in U(R)$.

Proposition 3.3. Let $I \unlhd R\left[X, X^{-1}\right]$ be an ideal, $J \unlhd R$, such that $I$ contains a doubly unitary polynomial. If $I+J\left[X, X^{-1}\right]=R\left[X, X^{-1}\right]$, then $(I \cap R)+J=R$.

Proof. Let us denote by $h_{1}$ a doubly unitary polynomial in $I$. Since $I+J\left[X, X^{-1}\right]=$ $R\left[X, X^{-1}\right]$, there exist $h_{2} \in I$ and $h_{3} \in J\left[X, X^{-1}\right]$ such that $h_{2}+h_{3}=1$. Let $g_{i}=X^{-\operatorname{ldeg}\left(h_{i}\right)} h_{i}$, for $i=1,2,3$. Since $X^{l} \in \sum_{i=1}^{3} g_{i} R[X]$, for some $l \geq 0$, and $g_{1} \equiv u \bmod X R[X]$, where $u \in U(R)$, we obtain that $\left\langle g_{1}, g_{2}, g_{3}\right\rangle=\langle 1\rangle$ in $R[X]$. By [8], Lemma 2], we obtain $\left(\left\langle g_{1}, g_{2}\right\rangle \cap R\right)+J=R$. So $(I \cap R)+J=R$.

Theorem 3.4. Let $f=\left(f_{0}, \ldots, f_{n}\right) \in \operatorname{Um}_{n+1}\left(R\left[X, X^{-1}\right]\right)$, where $n \geq 2$. Assume that $f_{0}$ is a doubly unitary polynomial; then

$$
f \sim_{\mathrm{E}_{n+1}\left(R\left[X, X^{-1}\right]\right)}(1,0, \ldots, 0) .
$$

Proof. By (2.4),

$$
\begin{aligned}
f & \sim_{\mathrm{E}}\left(X^{-\operatorname{ldeg}\left(f_{0}\right)} f_{0}, X^{-\operatorname{ldeg}\left(f_{0}\right)} f_{1}, f_{2}, \ldots, f_{n}\right) \\
& \sim_{\mathrm{E}}\left(X^{-\operatorname{ldeg}\left(f_{0}\right)} f_{0}, X^{-\operatorname{ldeg}\left(f_{0}\right)+2 k} f_{1}, X^{2 k} f_{2}, \ldots, X^{2 k} f_{n}\right)=\left(g_{0}, \ldots, g_{n}\right)
\end{aligned}
$$

where $k \in \mathbb{N}$. For sufficiently large $k$, we obtain that $g_{0}, \ldots, g_{n} \in R[X]$. Clearly, $X^{l} \in \sum_{i=0}^{n} g_{i} R[X]$ for some $l \geq 0$. But $g_{0} \equiv u \bmod X R[X]$, where $u \in U(R)$. Then $X^{l} R[X]+g_{0} R[X]=R[X]$, so $g \in \operatorname{Um}_{n}(R[X])$. By (2.1), $g \sim_{\mathrm{E}} e_{1}$. 
Remark 3.5. Let $a=\left(a_{1}, \ldots, a_{n}\right) \in \operatorname{Um}_{n+1}(R)$, where $n \geq 2$. If

$$
a \sim_{\mathrm{E}_{n}(R / \mathrm{Nil}(R))} e_{1},
$$

then $a \sim_{\mathrm{E}_{n}(R)} e_{1}$.

Proposition 3.6. If $R$ is a zero-dimensional ring and $f=\left(f_{0}, \ldots, f_{n}\right) \in$ $\operatorname{Um}_{n+1}\left(R\left[X, X^{-1}\right]\right)$, where $n \geq 1$, then

$$
f \sim_{\mathrm{E}} e_{1} .
$$

Proof. We prove by induction on $\operatorname{deg} f_{0}+\operatorname{deg} f_{1}$. We may assume that $R$ is a reduced ring. Let $a=\mathrm{hc}\left(f_{0}\right)$ and $b=\operatorname{lc}\left(f_{0}\right)$. Assume that $a b \in U(R)$. Then by elementary transformations of the form

$$
f_{1}-X^{\operatorname{ldeg}\left(f_{1}\right)-\operatorname{ldeg}\left(f_{0}\right)} b^{-1} \operatorname{lc}\left(f_{1}\right) f_{0},
$$

we obtain that $f \sim_{E}\left(f_{0}, h_{1}, f_{2}, \ldots, f_{n}\right)$, where $\operatorname{ldeg}\left(h_{1}\right)>\operatorname{ldeg}\left(f_{0}\right)$. By elementary transformations of the form

$$
f_{1}-X^{\operatorname{hdeg}\left(f_{1}\right)-\operatorname{hdeg}\left(f_{0}\right)} a^{-1} \operatorname{hc}\left(f_{1}\right) f_{0},
$$

we obtain that $f \sim_{E}\left(f_{0}, g_{1}, f_{2}, \ldots, f_{n}\right)$, where $\operatorname{ldeg}\left(g_{1}\right) \geq \operatorname{ldeg}\left(f_{0}\right)$ and $\operatorname{hdeg}\left(g_{1}\right)<$ $\operatorname{hdeg}\left(f_{0}\right)$. So we may assume that $\operatorname{deg} f_{0} \leq \operatorname{deg} f_{1}$ and $a b \notin U(R)$. Assume that $a \notin U(R)$. We have $R a=R e$ for some idempotent $e$. Let $c=\operatorname{hc}\left(f_{1}\right)$. Since $e \in R a$, we may assume that $c \neq 0$ and that $c \in R(1-e)$. Note that

$$
\begin{gathered}
(1-e) f=\left(f_{0}(1-e), \ldots, f_{n}(1-e)\right) \in \operatorname{Um}_{n+1}\left(R(1-e)\left[X, X^{-1}\right]\right) \text { and } \\
e f=\left(f_{0} e, \ldots, f_{n} e\right) \in \operatorname{Um}_{n+1}\left(\operatorname{Re}\left[X, X^{-1}\right]\right) .
\end{gathered}
$$

By the inductive assumption, there are matrices

$$
A \in \mathrm{E}_{n+1}\left(R(1-e)\left[X, X^{-1}\right]\right), B \in \mathrm{E}_{n+1}\left(R e\left[X, X^{-1}\right]\right)
$$

so that $(1-e) f A=(1-e, 0, \ldots, 0)$ and ef $B=(e, 0, \ldots, 0)$. Let

$$
A=\prod_{s=1}^{k} \mathrm{E}_{i j}\left(h_{s}\right), B=\prod_{s=1}^{t} \mathrm{E}_{i j}\left(g_{s}\right)
$$

where

$$
\mathrm{E}_{i j}\left(h_{s}\right)=(1-e) I_{n+1}+h_{s} e_{i j}, \mathrm{E}_{i j}\left(g_{s}\right)=e I_{n+1}+g_{s} e_{i j}
$$

and $i \neq j \in\{1, \ldots, n+1\}, h_{s} \in R(1-e)\left[X, X^{-1}\right], g_{s} \in R e\left[X, X^{-1}\right]$. Let

$$
A^{\prime}=\prod_{s=1}^{k}\left(I_{n+1}+h_{s} e_{i j}\right), B^{\prime}=\prod_{s=1}^{t}\left(I_{n+1}+g_{s} e_{i j}\right) .
$$

Clearly, $(1-e) A^{\prime}=A, e B^{\prime}=B$ and $A^{\prime}, B^{\prime} \in \mathrm{E}_{n+1}\left(R\left[X, X^{-1}\right]\right)$. Let $C=A^{\prime} B^{\prime}$; then $C \in \mathrm{E}_{n+1}\left(R\left[X, X^{-1}\right]\right)$ and

$$
(1-e) C=(1-e) A^{\prime}(1-e) B^{\prime}=A(1-e) I_{n+1}=(1-e) A^{\prime}=A .
$$

Similarly, we have $e C=B$. Let $f C=\left(g_{0}, \ldots, g_{n}\right)=g$. Thus

$$
g_{0}(1-e)=1-e \text { and } g_{1} e=e \text {. }
$$

So

$$
\begin{gathered}
f \sim_{\mathrm{E}_{n+1}\left(R\left[X, X^{-1}\right]\right)}\left(g_{0}, \ldots, g_{n}\right) \sim_{\mathrm{E}_{n+1}\left(R\left[X, X^{-1}\right]\right)}\left(g_{0}+e, \ldots, g_{n}\right) \\
=\left(1+g_{0} e, \ldots, g_{n}\right) \sim_{\mathrm{E}_{n+1}\left(R\left[X, X^{-1}\right]\right)}\left(1+g_{0} e,-g_{0} e, \ldots, g_{n}\right) \sim_{\mathrm{E}_{n+1}\left(R\left[X, X^{-1}\right]\right)} e_{1} .
\end{gathered}
$$

Similarly, if $b \notin U(R)$, then $f \sim_{\mathrm{E}} e_{1}$. 
Proposition 3.7. If $R$ is a zero-dimensional ring, then

$$
\mathrm{SL}_{n}\left(R\left[X, X^{-1}\right]\right)=\mathrm{E}_{n}\left(R\left[X, X^{-1}\right]\right)
$$

for all $n \geq 2$.

Proof. Clearly, $\mathrm{E}_{n}\left(R\left[X, X^{-1}\right]\right) \subseteq \mathrm{SL}_{n}\left(R\left[X, X^{-1}\right]\right)$. Let $M \in \mathrm{SL}_{n}\left(R\left[X, X^{-1}\right]\right)$. By (3.6), we can perform suitable elementary transformations to bring $M$ to $M_{1}$ with first row $(1,0, \ldots, 0)$. Now a sequence of row transformations brings $M_{1}$ to

$$
M_{2}=\left(\begin{array}{cc}
1 & 0 \\
0 & M^{\prime}
\end{array}\right)
$$

where $M^{\prime} \in \mathrm{SL}_{n-1}\left(R\left[X, X^{-1}\right]\right)$. The proof now proceeds by induction on $n$.

Lemma 3.8. Let $\left(f_{0}, \ldots, f_{n}\right) \in \operatorname{Um}_{n+1}\left(R\left[X, X^{-1}\right]\right)$, where $n \geq 2$. Assume that $\mathrm{hc}\left(f_{0}\right)$ is invertible modulo $f_{0}$. Then

$$
f \sim_{\mathrm{E}}\left(f_{0}, g_{1}, \ldots, g_{n}\right)
$$

where $\operatorname{hdeg}\left(g_{i}\right)<\operatorname{hdeg}\left(f_{0}\right), \operatorname{ldeg}\left(g_{i}\right) \geq \operatorname{ldeg}\left(f_{0}\right)$, for all $1 \leq i \leq n$.

Proof. By (2.4), $f \sim_{\mathrm{E}}\left(f_{0}, X^{2 k} f_{1}, \ldots, X^{2 k} f_{n}\right)$ for all $k \in \mathbb{Z}$. So we may assume that $\operatorname{ldeg}\left(f_{i}\right)>\operatorname{ldeg}\left(f_{0}\right)$. Let $a=\operatorname{hc}\left(f_{0}\right)$. By (2.4) we have

$$
f \sim_{\mathrm{E}}\left(f_{0}, a^{2} f_{1}, \ldots, a^{2} f_{n}\right) .
$$

Using elementary transformations of the form

$$
a^{2} f_{i}-a X^{\mathrm{hdeg}\left(f_{i}\right)-\operatorname{hdeg}\left(f_{0}\right)} \mathrm{hc}\left(f_{i}\right) f_{0}
$$

we lower the degrees of $f_{i}$, for all $1 \leq i \leq n$, and obtain the required row.

Lemma 3.9. Let $R$ be a ring of dimension $d>0$ and

$$
f=\left(r, f_{1}, \ldots, f_{n}\right) \in \mathrm{Um}_{n+1}\left(R\left[X, X^{-1}\right]\right)
$$

where $r \in R, n \geq d+1$. Assume that for every ring $T$ of dimension $<d$ and $n \geq$ $\operatorname{dim}(T)+1$, the group $\mathrm{E}_{n+1}\left(T\left[X, X^{-1}\right]\right)$ acts transitively on $\operatorname{Um}_{n+1}\left(T\left[X, X^{-1}\right]\right)$. Then $f \sim_{\mathrm{E}\left(R\left[X, X^{-1}\right]\right)} e_{1}$.

Proof. Since $\operatorname{dim}(R / \mathcal{I}(r))<\operatorname{dim}(R)$ over $R / \mathcal{I}(r)$, we can complete $\left(f_{1}, \ldots, f_{n}\right)$ to a matrix in $\mathrm{E}_{n}\left(R / \mathcal{I}(r)\left[X, X^{-1}\right]\right)$. If we lift this matrix, we obtain that

$$
\begin{gathered}
\left(r, f_{1}, \ldots, f_{n}\right) \sim_{\mathrm{E}_{n+1}\left(R\left[X, X^{-1}\right]\right)}\left(r, 1+r w_{1}+h_{1}, \ldots, r w_{n}+h_{n}\right) \sim_{\mathrm{E}_{n+1}\left(R\left[X, X^{-1}\right]\right)} \\
\left(r, 1+h_{1}, \ldots, h_{n}\right)
\end{gathered}
$$

where $h_{i}, w_{i} \in R\left[X, X^{-1}\right]$ and $r h_{i}=0$ for all $1 \leq i \leq n$. Then

$$
f \sim_{\mathrm{E}_{n+1}\left(R\left[X, X^{-1}\right]\right)}\left(r-r\left(1+h_{1}\right), 1+h_{1}, \ldots, h_{n}\right) \sim_{\mathrm{E}_{n+1}\left(R\left[X, X^{-1}\right]\right)} e_{1} .
$$

Lemma 3.10. Let $R$ be a ring of dimension $d>0$ and

$$
f=\left(f_{0}, \ldots, f_{n}\right) \in \operatorname{Um}_{n+1}\left(R\left[X, X^{-1}\right]\right)
$$

such that $n \geq d+1, f_{0}=a g$ and $a^{t}=\mathrm{hc}\left(f_{0}\right)$, where $a \in R \backslash U(R), 0 \neq t \in \mathbb{N}$. Assume that for every ring $T$ of dimension $<d$ and $n \geq \operatorname{dim}(T)+1$, the group $\mathrm{E}_{n+1}\left(T\left[X, X^{-1}\right]\right)$ acts transitively on $\operatorname{Um}_{n+1}\left(T\left[X, X^{-1}\right]\right)$. Then $f \sim_{\mathrm{E}\left(R\left[X, X^{-1}\right]\right)} e_{1}$. 
Proof. We prove by induction on the number $M$ of non-zero coefficients of the polynomial $f_{0}$ that $f \sim_{\mathrm{E}} e_{1}$. If $M=1$, then $f_{0}=r X^{m}$ where $r \in R, m \in$ $\mathbb{Z}$. By (2.4), $f \sim_{\mathrm{E}}\left(r, X^{-m} f_{1}, f_{2}, \ldots, f_{n}\right)$. So by (3.9), we obtain that $f \sim_{\mathrm{E}} e_{1}$. Assume now that $M>1$. Let $S$ be the multiplicative subset of $R$ generated by $a, b$, where $b=\operatorname{lc}(g)$; i.e., $S=\left\{a^{k_{1}} b^{k_{2}} \mid k_{1}, k_{2} \in \mathbb{N}\right\}$. By the inductive step, with respect to the ring $R / a b R$, we obtain from $f$ a row $\equiv(1,0, \ldots, 0) \bmod a b R\left[X, X^{-1}\right]$. We can perform such transformations so that at every stage the row contains a doubly unitary polynomial in $R_{S}\left[X, X^{-1}\right]$. Indeed, if we have to perform, e.g., the elementary transformation

$$
\left(g_{0}, \ldots, g_{n}\right) \rightarrow\left(g_{0}, g_{1}+h g_{0}, \ldots, g_{n}\right)
$$

and $g_{1}$ is a doubly unitary polynomial in $R_{S}\left[X, X^{-1}\right]$, then we replace this elementary transformation by the two transformations

$$
\begin{gathered}
\left(g_{0}, \ldots, g_{n}\right) \rightarrow\left(g_{0}+a b X^{m} g_{1}+a b X^{k} g_{1}, g_{1}, \ldots, g_{n}\right) \\
\rightarrow\left(g_{0}+a b X^{m} g_{1}+a b X^{k} g_{1}, g_{1}+h\left(g_{0}+a b X^{m} g_{1}+a b X^{-k} g_{1}\right), \ldots, g_{n}\right)
\end{gathered}
$$

where $m>\operatorname{hdeg}\left(g_{0}\right), k<\operatorname{ldeg}\left(g_{0}\right)$. So we may assume that

$$
\left(f_{0}, \ldots, f_{n}\right) \equiv(1,0, \ldots, 0) \bmod a b R\left[X, X^{-1}\right]
$$

and $f_{0}$ is a doubly unitary polynomial in $R_{S}\left[X, X^{-1}\right]$. By (3.8), we may assume that $\operatorname{hdeg}\left(f_{i}\right)<\operatorname{hdeg}\left(f_{0}\right), \operatorname{ldeg}\left(f_{i}\right) \geq \operatorname{ldeg}\left(f_{0}\right)$.

We prove that $f$ can be transformed by elementary transformation into a row with one constant entry. We use an argument similar to that in the proof of [ [ $[\overline{5}$, Theorem 5].

Assume that the number of coefficients of $f_{2}, \ldots, f_{n}$ is $\geq 2(n-1)$. Since $d>0$, we obtain that $2(n-1) \geq d+1$. Let $a_{1}, \ldots, a_{t}$ be the coefficients of $f_{2}, \ldots, f_{n}$ and $J=\frac{a_{1}}{1} R_{S}+\cdots+\frac{a_{t}}{1} R_{S}$. Let $I=R_{S}\left[X, X^{-1}\right] f_{0}+R_{S}\left[X, X^{-1}\right] f_{1}$. Since $I+J\left[X, X^{-1}\right]=R_{S}\left[X, X^{-1}\right]$ and $f_{0}$ is doubly unitary in $R_{S}\left[X, X^{-1}\right]$, by (3.3), we obtain that $\left(I \cap R_{S}\right)+J=R_{S}$. So $\left(\frac{f_{0} h_{0}+f_{1} h_{1}}{s}\right)+\frac{r_{1}}{s_{1}} \frac{a_{1}}{1}+\cdots+\frac{r_{t}}{s_{t}} \frac{a_{t}}{1}=\frac{1}{1}$, where $h_{0}, h_{1} \in R\left[X, X^{-1}\right]$ and $r_{i} \in R, s, s_{i} \in S$ for all $1 \leq i \leq t$. This means that $\left(\frac{f_{0} h_{0}+f_{1} h_{1}}{1}, \frac{a_{1}}{1}, \ldots, \frac{a_{t}}{1}\right) \in \mathrm{Um}_{t+1}\left(R_{S}\right)$. By (2.3) , there exist $s \in S$ and $b_{1}, \ldots, b_{t} \in R$, such that

$$
s \in\left(a_{1}+b_{1}\left(f_{0} h_{0}+f_{1} h_{1}\right)\right) R+\cdots+\left(a_{t}+b_{t}\left(f_{0} h_{0}+f_{1} h_{1}\right)\right) R .
$$

Using elementary transformations, we may assume that $J=R_{S}$. By (3.2), the ideal $\left\langle f_{0}, f_{2}, \ldots, f_{n}\right\rangle$ contains a polynomial $h$ such that $a^{k_{1}} b^{k_{2}}=\mathrm{hc}(h)$ and hdeg $(h)=$ $\operatorname{hdeg}\left(f_{0}\right)-1, \operatorname{ldeg}(h) \geq \operatorname{ldeg}\left(f_{0}\right)$ where $k_{1}, k_{2} \in \mathbb{N}$. Let $r=\operatorname{hc}\left(f_{1}\right)$, so

$$
f \sim_{\mathrm{E}}\left(f_{0}, a^{2 k_{1}} b^{2 k_{2}} f_{1}, f_{2}, \ldots, f_{n}\right) \sim_{\mathrm{E}}\left(f_{0}, a^{2 k_{1}} b^{2 k_{2}} f_{1}+\left(1-a^{k_{1}} b^{k_{2}} r\right) h, f_{2}, \ldots, f_{n}\right) .
$$

Then we may assume that $a^{k_{1}} b^{k_{2}}=\mathrm{hc}\left(f_{1}\right)$. By the proof of Lemma (3.8), we can decrease the $\operatorname{hdeg}\left(f_{i}\right)$ for all $2 \leq i \leq n$.

Repeating the argument above, we obtain that

$$
f \sim_{\mathrm{E}}\left(r X^{m}, g_{1}, \ldots, g_{n}\right) \sim_{\mathrm{E}}\left(r, g_{1} X^{-m}, g_{2}, \ldots, g_{n}\right)
$$

where $r \in R, m \in \mathbb{Z}, g_{1}, \ldots, g_{n} \in R\left[X, X^{-1}\right]$. By (3.9), $f \sim_{\mathrm{E}} e_{1}$.

Lemma 3.11. Let $R$ be a ring of dimension $d>0$ and

$$
f=\left(f_{0}, \ldots, f_{n}\right) \in \operatorname{Um}_{n+1}\left(R\left[X, X^{-1}\right]\right)
$$


such that $n \geq d+1, f_{0}=c g$ and $c^{t}=\operatorname{lc}\left(f_{0}\right)$, where $c \in R \backslash U(R), 0 \neq t \in \mathbb{N}$. Assume that for every ring $T$ of dimension $<d$ and $n \geq \operatorname{dim}(T)+1$, the group $\mathrm{E}_{n+1}\left(T\left[X, X^{-1}\right]\right)$ acts transitively on $\mathrm{Um}_{n+1}\left(T\left[X, X^{-1}\right]\right)$. Then $f \sim_{\mathrm{E}\left(R\left[X, X^{-1}\right]\right)} e_{1}$.

Proof. By making the change of variable: $X \rightarrow X^{-1}$ and Proposition (3.10), we obtain that $f \sim_{\mathrm{E}\left(R\left[X, X^{-1}\right]\right)} e_{1}$.

Theorem 3.12. Let $R$ be a ring of dimension $d$ and $n \geq d+1$. Then $\mathrm{E}_{n+1}\left(R\left[X, X^{-1}\right]\right)$ acts transitively on $\operatorname{Um}_{n+1}\left(R\left[X, X^{-1}\right]\right)$.

Proof. Let $f=\left(f_{0}, \ldots, f_{n}\right) \in \operatorname{Um}_{n+1}\left(R\left[X, X^{-1}\right)\right)$. We prove the theorem by induction on $d$; we may assume that $R$ is a reduced ring. If $d=0$, by (3.6), we are done. Assume that the theorem is true for the dimensions $0,1, \ldots, d-1$, where $d>0$. We prove by induction on the number $N$ of non-zero coefficients of the polynomials $f_{0}, \ldots, f_{n}$, that $f \sim_{\mathrm{E}} e_{1}$ if $\operatorname{dim} R=d$. Starting with $N=1$, let $N>1$. Let $a=\mathrm{hc}\left(f_{0}\right)$ and $c=\operatorname{lc}\left(f_{0}\right)$. If $a c \in U(R)$, then by (3.4), we are done. Otherwise, assume that $a \notin U(R)$. By the inductive step, with respect to the ring $R / a R$, we obtain from $f$ a row $\equiv(1,0, \ldots, 0)$ mod $a R\left[X, X^{-1}\right]$ using elementary transformations. We can perform such transformations so that at every stage the row contains a polynomial $g \in R\left[X, X^{-1}\right]$ such that $h c(g)=a^{t}$, where $t \in \mathbb{N}$. Indeed, if we have to perform, e.g., the elementary transformation

$$
\left(g_{0}, \ldots, g_{n}\right) \rightarrow\left(g_{0}, g_{1}+h g_{0}, \ldots, g_{n}\right)
$$

and $\mathrm{hc}\left(g_{1}\right) \in U\left(R_{a}\right)$, then we replace this elementary transformation by the two transformations:

$\left(g_{0}, \ldots, g_{n}\right) \rightarrow\left(g_{0}+a X^{m} g_{1}, g_{1}, \ldots, g_{n}\right) \rightarrow\left(g_{0}+a X^{m} g_{1}, g_{1}+h\left(g_{0}+a X^{m} g_{1}\right), \ldots, g_{n}\right)$

where $m>\operatorname{hdeg}\left(g_{0}\right)$.

So we have $f_{0}=a g$, and $a^{t}=\mathrm{hc}\left(f_{0}\right)$, where $0 \neq t \in \mathbb{N}$. By (3.10), $f \sim_{\mathrm{E}} e_{1}$. Similarly, if $c \notin U(R)$, by (3.11) we obtain that $f \sim_{\mathrm{E}} e_{1}$.

Corollary 3.13. For any ring $R$ with Krull dimension $\leq d$, all finitely generated stably free modules over $R\left[X, X^{-1}\right]$ of rank $>d$ are free.

The following conjecture is the analogue of Conjecture 8 of [7] in the Laurent case:

Conjecture 3.14. For any ring $R$ with Krull dimension $\leq d$, all finitely generated stably free modules over $R\left[X_{1}^{ \pm 1}, \ldots, X_{k}^{ \pm 1}, X_{k+1}, \ldots, X_{n}\right]$ of rank $>d$ are free.

\section{ACKNOWLEDGMENT}

The author would like to thank Professor Moshe Roitman, his M.Sc. thesis advisor, for his interest in this project.

\section{REFERENCES}

1. H. Bass, Libération des modules projectifs sur certains anneaux de polynômes, Sém. Bourbaki 1973/74, exp. 448, Lecture Notes in Math., vol. 431, Springer-Verlag, Berlin and New York, 1975, 228-254. MR0472826 (57:12516)

2. T. Coquand, H. Lombardi, C. Quitté, Generating non-noetherian modules constructively, Manuscripta Math. 115 (2004) 513-520. MR2103665 (2005h:13014)

3. T. Coquand, H. Lombardi, M. F. Roy, An elementary characterization of Krull dimension, From sets and types to analysis and topology: towards practicable foundations for constructive mathematics, Oxford University Press, 2005. MR2188647 (2007a:54023) 
4. R. A. Rao, An elementary transformation of a special unimodular vector to its top coefficient vector, Proc. Amer. Math. Soc. 93 (1985), no. 1, 21-24. MR766519 (86a:13011)

5. M. Roitman, On stably extended projective modules over polynomial rings, Proc. Amer. Math. Soc. 97 (1986), no. 4, 585-589. MR845969 (87f:13007)

6. A. A. Suslin, On the structure of the special linear group over polynomial rings, Izv. Math. 11 (1977), no. 2, 221-238.

7. I. Yengui, Stably free modules over $\mathbf{R}[X]$ of rank $>\operatorname{dim} \mathbf{R}$ are free, Math. Comp. 80 (2011), 1093-1098.

8. I. Yengui, The Hermite ring conjecture in dimension one, J. Algebra, 320 (2008), no. 1, 437-441. MR 2417998 (2009g:16019)

Department of Mathematics, University of Haifa, Mount Carmel, Haifa 31905, Israel E-mail address: abedelfatah@gmail.com 